\section{FRI0117 TOCILIZUMAB USE DIRECTLY AFTER SDMARD FAILURE SHOWS HIGHER RESPONSE RATES AND A LONGER TREATMENT DURATION}

F. Behrens $^{1}$, W.A. Biewer ${ }^{2}$, G.-R. Burmester ${ }^{3}$, M. Feuchtenberger ${ }^{4}$, J.-P. Flacke ${ }^{5}$, M. Hofmann ${ }^{6}$, P. Kästner ${ }^{7}$, H. Kellner ${ }^{8}$, T. Klopsch ${ }^{9}$, C. Kühne ${ }^{10}$, H.-P. Tony ${ }^{11}$, C. Amberger ${ }^{12} .^{1} \mathrm{CIRI}$ am Klinikum der Johann Wolfgang Goethe-Universität, Rheumatologie, Fraunhofer Institute for Molecular Biology and Applied Ecology IME, Project Group Translational Medicine and Pharmacology TMP, Frankfurt Main; ${ }^{2}$ Rheumatologische Schwerpunktpraxis Saarbrücken, Saarbrücken; ${ }^{3}$ Charité - Universitätsmedizin Berlin, Medizinische Klinik mit Schwerpunkt Rheumatologie und klinische Immunologie, Berlin; ${ }^{4}$ Rheumatologie/Klinische Immunologie, Kreiskliniken Altötting-Burghausen, Burghausen; ${ }^{5}$ Roche Pharma AG, GrenzachWyhlen; ${ }^{6}$ Chugai Pharma AG, Frankfurt/Main; ${ }^{7}$ MVZ Ambulantes Rheumazentrum Erfurt, Erfurt; ${ }^{8}$ Schwerpunktpraxis für Rheumatologie und Gastroenterologie, München; ${ }^{9}$ Rheumatologische Praxis, Neubrandenburg; ${ }^{10}$ Praxis, Haldensleben; ${ }^{11}$ Universitätsklinikum Würzburg, Medizinische Klinik und Poliklinik II, Schwerpunkt Rheumatologie und klinische Immunologie, Würzburg; ${ }^{12}$ Rheumatologische Gemeinschaftspraxis Dr. Pick/Dr. Amberger, Bad Neuenahr, Germany

Background: The non-interventional study (NIS) ARATA (NCT02251860) observes the clinical effectiveness and safety of subcutaneous Tocilizumab (TCZ) s.c. treatment under routine conditions over a 2 year period.

Objectives: In this interim analysis, the patients were subgrouped according to their pretreatment: (I) pretreated exclusively with SDMARD or (II) also pretreated with bDMARD.

Methods: TCZ-naive patients (Pts) ( $\geq 18$ years) with RA, who receive TCZ s.c. treatment, could be included in the study since 2014. Demographic and diseasespecific characteristics, the progression of the disease (rheumatoid activity scores), concomitant medications, adverse events (AE) and patient questionnaires were documented.

Results: In this interim analysis (reporting date 01-FEB-2017), the data of 912 Pts were evaluated. 319 Pts (35\%) were pretreated exclusively with sDMARD and 585 Pts $(64.8 \%)$ were also pretreated with bDMARD. The main reason for a switch to TCZ s.c. was lacking effectiveness of the pretreatment.

In comparison, patients exclusively pretreated with sDMARD demonstrated a shorter median disease duration ( 6 vs. 9 years), TCZ s.c. was applied at BL more rarely in combination with $\mathrm{MTX}(23.5 \% \mathrm{vs} .35 .6 \%)$ and more rarely with glucocorticoids $(59.2 \%$ vs. $69.5 \%)$. The comorbidity rates were comparable in both groups. However, patients exclusively pretreated with SDMARD suffered half as often from osteoporosis $(9.1 \%$ vs. $21.2 \%)$. Patients Exclusively pretreated with sDMARD had a longer median treatment duration (380.5 vs. 341.0 days) and a higher retention rate to week $52(78.9 \%$ vs. $70.5 \%)$. The effectiveness of the TCZ s.c. treatment was examined with 831 patients. More patients with exclusively sDMARD pretreatment achieved a DAS28 remission and remained in remission (figure 1). The change from $\mathrm{BL}$ for the $\mathrm{CDAl}$ and Dcrit response was higher in the sDMARD subgroup. No new safety signals and no differences between the subgroups for all safety parameters were observed.

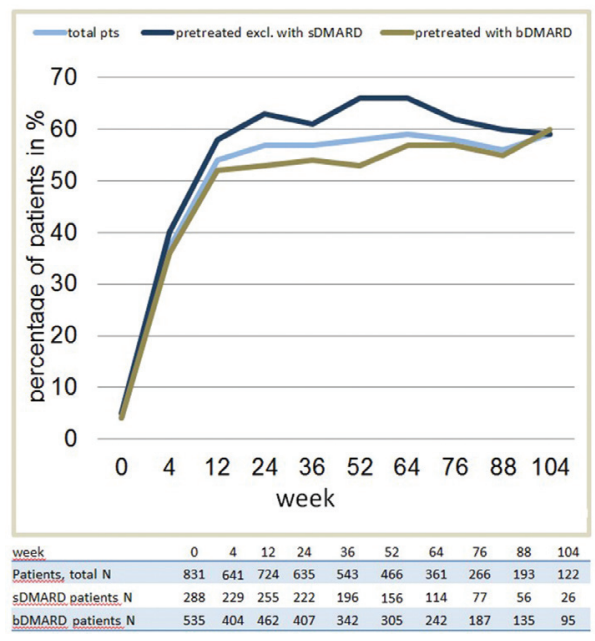

Abstract FRI0117 - Figure 1. Clinical remission (DAS28- ESR <2.6) after pretreatment $(\mathrm{N}=831)$. DAS28-ESR, Disease activity score 28 -erythrocyte sedimentation rate.
Conclusions: The results of the third interim analysis of the NIS ARATA confirm the efficacy of TCZ s.c. observed in the approval trials in clinical practice. A fast and effective reduction of disease activity in the treated RA patients as well as a lasting improvement in all RA progression parameters collected was observed. In case of the earlier application of TCZ s.c., directly after SDMARD failure, higher response rates and a longer retention of the treatment were observed.

Disclosure of Interest: F. Behrens Grant/research support from: Abbvie, Pfizer, Roche, Chugai, Janssen, Novartis, Consultant for: Abbvie, Pfizer, Roche, Chugai, UCB, BMS, Celgene, MSD, Novartis, Biotest, Janssen, Genzyme, Lilly, Boehringer, Sandoz, W. A. Biewer: None declared, G.-R. Burmester Consultant for: Lilly, Pfizer, Sanofi, Roche, M. Feuchtenberger Consultant for: MSD, Roche, Abbvie, Chugai, Pfizer, UCB, J.-P. Flacke Employee of: Roche Pharma AG, M. Hofmann Employee of: Chugai Pharma Europe Ltd., P. Kästner: None declared, H. Kellner Consultant for: Roche, T. Klopsch: None declared, C. Kühne Consultant for: Celgene, Roche, Pfizer, Novartis, BMS, H.-P. Tony Consultant for: Roche Pharma, Abbvie, BMS, Chugai, Janssen, Novartis, Sanofi, Lilly, Speakers bureau: Roche Pharma, Abbvie, BMS, Chugai, Janssen, Novartis, Sanofi, Lilly, C. Amberger Consultant for: Chugai, AbbVie, Celgene, MSD, Pfizer, BMS, HexaL DOI: 10.1136/annrheumdis-2018-eular.2166

\section{FRI0118 DEKAVIL (F8IL10) - UPDATE ON THE RESULTS OF CLINICAL TRIALS INVESTIGATING THE IMMUNOCYTOKINE IN PATIENTS WITH RHEUMATOID ARTHRITIS}

M. Galeazzi ${ }^{1}$, G. Sebastiani ${ }^{2}$, R. Voll ${ }^{3}$, O. Viapiana ${ }^{4}$, J. Dudler ${ }^{5}$, P. Zufferey ${ }^{6}$, E. Selvi ${ }^{1}$, S. Finzel ${ }^{3}$, F.S. Bootz ${ }^{7}$, D. Neri ${ }^{8}$, on behalf of Dekavil Study Group. ${ }^{1}$ University Hospital Siena, Siena; ${ }^{2}$ Rheumatology, San Camillo-Forlanini Hospital, Rome, Italy; ${ }^{3}$ University Medical Center Freiburg, Freiburg, Germany; ${ }^{4}$ University Hospital Verona, Verona, Italy; ${ }^{5}$ Cantonal Hospital Fribourg, Fribourg: ${ }^{6} \mathrm{CHUV}$, Lausanne; ${ }^{7}$ Philochem Ag, Philogen Group, Otelfingen, Switzerland; ${ }^{8}$ Philogen SpA, Siena, Italy

Background: The targeted pharmacodelivery of cytokines by means of antibodyfusion proteins aims at selective accumulation of the active compound at the site of disease while sparing healthy tissues. Dekavil (F8IL10) is a fully human fusion protein composed of the vascular targeting antibody F8 fused to the cytokine interleukin-10. A phase 1 dose escalation study in patients with rheumatoid arthritis (RA) has recently been completed. Dekavil is currently under investigation in a phase 2 clinical study for the treatment of RA.

Objectives: The phase 1 study investigated safety, tolerability and the maximum tolerated dose of Dekavil when administered in combination with methotrexate (MTX). The currently ongoing phase 2 (in patients with active RA despite MTX therapy who had failed anti-TNF treatment) measures the mean change from baseline in DAS28-CRP between F8IL10 and placebo arms as primary endpoint. Methods: The target population of both RA studies comprises patients with active RA despite MTX therapy who had failed anti-TNF treatment. Dekavil is administered once weekly for 8 consecutive weeks by s.c. injection in combination with a fixed dose of MTX (10-25 mg). The recently completed phase 1 study investigated escalating doses of Dekavil $(6-600 \mu \mathrm{g} / \mathrm{kg}+\mathrm{MTX})$. The ongoing randomised multicenter, double-blind, placebo-controlled phase 2 trial assesses therapeutic activity in two treatment groups (Dekavil 30 or $160 \mu \mathrm{g} / \mathrm{kg}$ plus MTX) and one placebo group (placebo plus MTX).

Results: Dekavil was well tolerated up to the highest investigated dose $(600 \mu \mathrm{g} /$ $\mathrm{kg}$ ) and an MTD was not reached. In 34 out of 35 patients treated in the phase 1 study no SAEs and no SUSARs were reported. One subject (cohort 9, $450 \mu \mathrm{g} / \mathrm{kg}$ ) experienced a DLT (G2 purpura), which was accompanied by an SAE (G2 dyspnea, not drug related). The patient fully recovered within one week following corticosteroid administration. The most frequently observed adverse event was mild (G1) injection site reaction and occurred in $60 \%$ of the patients. Furthermore, two cases of drug related anaemia (G3 and G2; $160 \mu \mathrm{g} / \mathrm{kg}$ and $450 \mu \mathrm{g} / \mathrm{kg}$, respectively) were reported in this study. All adverse reactions resolved completely. After 4 cycles of treatment, $36.4 \%$ of patients (12/33) exhibited ACR responses. The fraction of responses increased to $45.8 \%(11 / 24)$ after 8 cycles. Two patients achievedan ACR70 for more than 12 months after the last drug administration.

As of January 2018, 25 patients have been treated in the phase 2 clinical study and neither SUSARs nor treatment-related deaths were recorded. Immunogenicity assessment is completed for the first 20 study subjects did not reveal an antibody response specific to F8IL10. An interim read-out after 45 patients will provide a more thorough understanding of the therapeutic activity of F8IL10. 


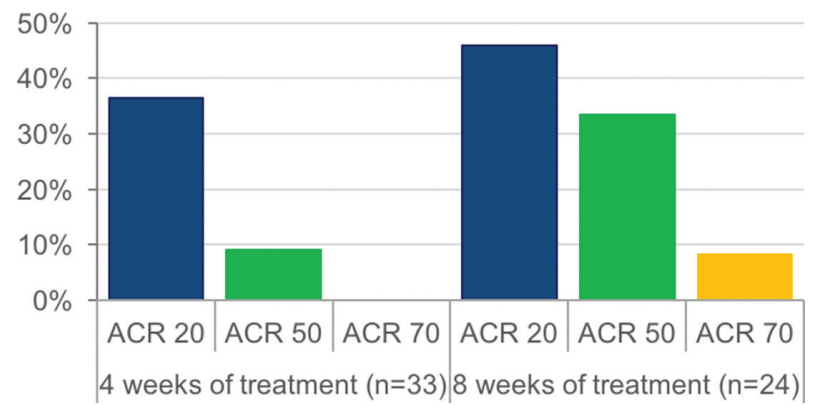

Abstract FRI0118 - Figure 1. ACR responses after 4 and after 8 weeks of Dekavil treatment in the phase 1 study population including all dose levels $(6-600 \mu \mathrm{g} / \mathrm{kg})$

Conclusions: The data obtained in the population studied to date suggest that Dekavil may be a safe and well tolerated novel therapeutic for the potential treatment of RA.

Disclosure of Interest: M. Galeazzi: None declared, G. Sebastiani: None declared, R. Voll: None declared, O. Viapiana: None declared, J. Dudler: None declared, P. Zufferey: None declared, E. Selvi: None declared, S. Finzel: None declared, F. Bootz Employee of: Philogen Group (Sponsor of the study), D. Neri Shareholder of: Philogen Group (Sponsor of the study)

DOI: 10.1136/annrheumdis-2018-eular.5550

\section{FRI0119 EFFECT OF DISCONTINUING TNF INHIBITORS DURING PREGNANCY ON THE COURSE OF RHEUMATOID ARTHRITIS AND JUVENILE IDIOPATHIC ARTHRITIS}

F. Förger ${ }^{1}$, G. Bandoli ${ }^{2}$, Y. Luo ${ }^{2}$, L. Robinson ${ }^{2}$, D.L. Johnson², C.D. Chambers ${ }^{2}$. ${ }^{1}$ Rheumatology, Immunology, Allergology, Inselspital UniversitäTsspital Bern, Bern, Switzerland; ${ }^{2}$ Department of Pediatrics, Medicine, and Family and Preventive Medicine, University of California San Diego, San Diego, USA

Background: Treatment changes at early pregnancy can be followed by a disease worsening. ${ }^{1}$

Objectives: To investigate whether the discontinuation of tumour necrosis factor inhibitors (TNFi) use during pregnancy is associated with any changes of disease activity at the third trimester in women with rheumatoid arthritis (RA) and juvenile idiopathic arthritis (JIA)

Methods: A prospective cohort study was conducted using the Organisation of Teratology Information Specialists (OTIS) Autoimmune Diseases in Pregnancy Project in the U.S. and Canada. Pregnant women with RA and JIA were enrolled between 2005 and 2017. Information about medication and disease activity were collected by telephone-based interviews prior to gestational week 20 and at gestational week 32. Disease activity was assessed by the Health Assessment Questionnaire Disability Index (HAQ-DI), the patient's pain scale and the patient's global scale. The composite tool Patient Activity Scale (PAS) was calculated in retrospect.

Results: In the OTIS cohort, data were available for 490 women of whom 397 had RA and 93 had JIA. Of all patients, $323(65.9 \%)$ used TNFi during pregnancy of whom $122(24.9 \%)$ patients discontinued TNFi before gestational week 20 (the mean time of discontinuation was gestational week $6(S D \pm 5.03))$ and 201 (41.0\%) used TNFi beyond week 20. There were 167 (34.1\%) patients not taking TNFi during pregnancy. At the time of enrollment, disease activity was low to minimal in $357(72.9 \%)$ patients as defined by PAS scores below 3.7 .

From the first to the third trimester, women using TNFi beyond week 20 showed a decrease of the PAS scores ( $p=0.02$, figure 1$)$ whereas women not using TNFi and those discontinuing TNFi before gestational week remained stable.

The univariate regression analysis, but not the adjusted model, revealed that TNFi use beyond week 20 was associated with improved HAQ scores at the third trimester (coefficient $\mathrm{B}-0.142,95 \% \mathrm{Cl}-0.258$ to -0.026 ) and with improved PAS scores (coefficient $\mathrm{B}-0.423,95 \% \mathrm{Cl}-0.843$ to -0.002 ). However, the various TNFi treatment modes during pregnancy were not associated with any minimum clinically important difference at the third trimester.

When selecting for 58 patients with active disease (PAS score $>3.71$ ) at the first trimester, the discontinuation of TNFi before gestational week 20 was not associated with any clinically important worsening of the disease at the third trimester.

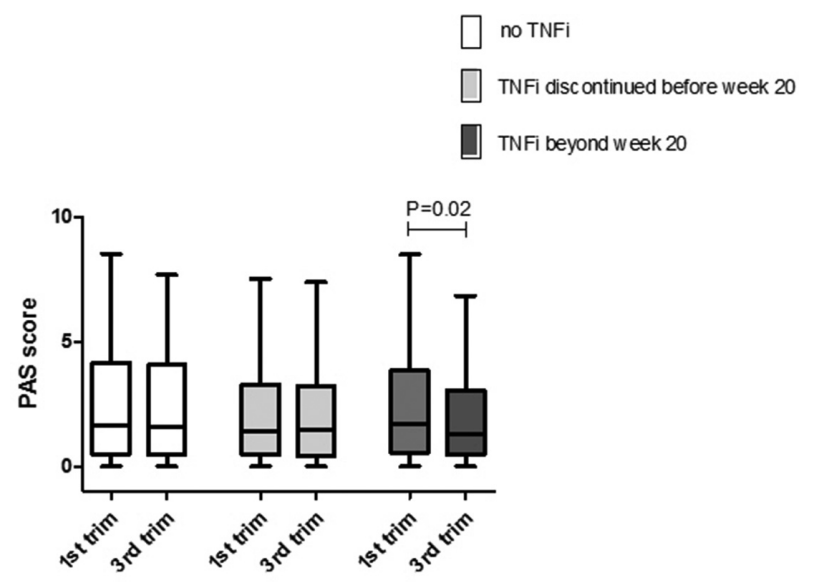

Conclusions: In patients with RA and JIA who enter pregnancy with well controlled disease, the discontinuation of TNFi before gestational week 20 is possible without a risk of disease flares at the third trimester.

\section{REFERENCE:}

[1] van den Brandt S, Zbinden A, Baeten D, Villiger PM, Ostensen M, Forger F. Risk factors for flare and treatment of disease flares during pregnancy in rheumatoid arthritis and axial spondyloarthritis patients. Arthritis research \& therapy. 2017;19(1):64.

Disclosure of Interest: None declared DOI: 10.1136/annrheumdis-2018-eular.5138

\section{FRI0120 EVALUATION OF RITUXIMAB, TOCILIZUMAB AND ABATACEPT IN A FRENCH MULTICENTER RHUPUS COHORT}

G. Laustriat ${ }^{1}$, O. Fogel ${ }^{2}$, D. Wendling ${ }^{3}$, S. Ottaviani ${ }^{4}$, S. Guignard ${ }^{5}$, J. Morel ${ }^{6}$, J. E. Gottenberg ${ }^{7}$ A. Cantagrel ${ }^{8}$, on behalf of CRI (Club Rhumatisme Inflammation). ${ }^{1}$ Rheumatology Department, Chu Toulouse Purpan, Toulouse; ${ }^{2}$ Hopital COCHIN, PARIS; ${ }^{3}$ Rheumatology Department, CHRU de Besançon, Besancon; ${ }^{4} A P-H P$, Hôpital Bichat-Claude Bernard, Service de Rhumatologie, Paris; ${ }^{5}$ Rheumatology Department, AP- HP, Hopital Henri Mondor, Creteil; ${ }^{6}$ Rheumatology Department, Chu Montpellier, Montpellier, ${ }^{7}$ Rheumatology Department, CHU Strasbourg, STRASBOURG: ${ }^{8}$ Chu Toulouse Purpan, Toulouse, France

Background: Rhupus, a combination of Rheumatoid Arthritis (RA) and Systemic Lupus Erythematosus, is a rare entity. Existing epidemiological and therapeutic data are limited.

Objectives: The aim of this study was to describe the therapeutic impact and safety of three biologic therapies: Rituximab, Tocilizumab and Abatacept in a french Rhupus cohort.

Methods: We have set up a transverse observational retrospective and multicentric study. To be included in the cohort, patients had to present an overlap syndrome fulfilling criterias for rheumatoid arthritis and lupus, and to be treated at least by one of these three therapies. Enrollment has been made with a file available on the CRI website and the analysis of the French RA registers (AIR, REGATE and ORA). Primary endpoint was the median time in therapeutic maintenance for each biological agent.

Results: Forty patients from fifteen rheumatologic centres were included. The main demographic data for these patients are given in table 1 . Thirty of them received a treatment with Rituximab, twelve with Tocilizumab and seven with Abatacept. Nine patients received 2 biologics at two different times of the disease. The medians of therapeutic maintenance were 82 months with Rituximab, 48 with Tocilizumab and 55 with Abatacept. The detailed analysis of clinical and biologic parameters revealed differences in effectiveness between therapies: corticosteroid doses decreased more in Rituximab group, VAS activity decreased more in Abatacept group, CRP decreased more in Tocilizumab group. Safety of biologics was similar to the data in literature for RA patients. 